\title{
Evaluation of Drain Output Monitoring in Surgical Ward
}

\author{
Mohammad Miah, Ramprasad Rajebhosale, Cindy Cleto, Daniel Centea, Rabia Yusuf, \\ Prabhu Ravi, Rajesh Paul, Najam Husain, Pradeep Thomas
}

Queens Hospital Burton, Burton on Trent, DE13 0RB, Staffordshire, UK

Email:mohammad.miah1@nhs.net

How to cite this paper: Miah, M., Rajebhosale, R., Cleto, C., Centea, D., Yusuf, R., Ravi, P., Paul, R., Husain, N. and Thomas, P. (2020) Evaluation of Drain Output Monitoring in Surgical Ward. Open Access Library Journal, 7: e6497.

https://doi.org/10.4236/oalib.1106497

Received: June 3, 2020

Accepted: July 18, 2020

Published: July 21, 2020

Copyright (C) 2020 by author(s) and Open Access Library Inc.

This work is licensed under the Creative

Commons Attribution International

License (CC BY 4.0).

http://creativecommons.org/licenses/by/4.0/

\begin{abstract}
The output from surgical drains often guides management as drains are commonly not removed until the wound bed drains $<50 \mathrm{mls}$ a day. A drain must be emptied and measured at least once every shift and cleaned using sterile technique according to Trust protocol. Failure to have a clear 24-hour output often results in drains that could be safely removed staying in-situ for a further day, increasing the risk of unnecessary pain and infection. Our purpose was to identify the number of patients undergoing major surgery or advised by the surgeon do they have drain output (DO) monitoring in place. Prospective data were collected over 2 weeks by patients' record review and bedside examination. 8 patients had drain in situ during this period. Drain output was monitored in 7 patients. Only 4 patients had a plan in place for drain output monitoring. Monitoring the output from surgical drains is important part of post-operative care. Appropriate drain output monitoring would improve patient safety, the efficiency of patient discharge and the stress of the surgical ward round.
\end{abstract}

\section{Subject Areas \\ Clinical Trials}

\section{Keywords}

Surgical Drains, Drain Output Monitoring, DO Monitoring

\section{Introduction}

Monitoring the output from surgical drains is an important part of post-operative care and is often undertaken poorly. Clear daily totals are re- 
quired to accurately assess a patient's fluid balance and are often pertinent to the timing of drain removal [1] [2]. The output from surgical drains often guides management as drains are commonly not removed until the wound bed drains < $50 \mathrm{mls}$ a day. A drain must be emptied and measured at least once every shift and cleaned using sterile technique according to Trust protocol. Failure to have a clear 24-hour output often results in drains that could be safely removed staying in-situ for a further day, increasing the risk of unnecessary pain and infection. As middle grade Doctor of Surgery, we noticed we were having regular difficulty in finding a daily drain output as there was no uniformity in the location or quality of documentation. Reasons for this are multifactorial, but a significant issue has been the shift in hospital documentation towards "early warning systems" and algorithmic systems of observations [3] that do not include output from surgical drains and thus are unlikely to afford sufficient space on these charts. A similar problem is faced by fluid charts. These typically require some degree of adaptation to account for multiple drains, as they are present with complex surgery patients. This problem added both time and frustration to the morning ward round as well as increasing the risk to patients associated with delaying drain removal. The drain output should always be accurately measured and appropriately recorded. The recommendation in PATIENT journal about Surgical drain and its indications, Management and Removal [(evidence-based information), Last edited 27 Jul. 2015, Certified by The Information Standard] is as follows:

- Monitor changes in character or volume of fluid. Identify any complications resulting in leaking fluid (particularly, for example, bile or pancreatic secretions) or blood.

- Use measurements of fluid loss to assist intravenous replacement of fluids.

\section{Methods and Materials}

Output of surgical drains should be monitored for procedural complications such as bleeding and as such, the type and location of drains needs to be quickly and accurately established. The minimum daily data set to be documented from each drain should therefore be the location and type of each drain, its 24-hour output, and the nature of the fluid draining, i.e. serous, serosanguinous, or seropurulent. Our purpose was to identify the number of patients undergoing major surgery or advised by the surgeon do they have drain output (DO) monitoring in place. Prospective data about drain output monitoring including basic demographic data were collected over 2 weeks by patients' record review and bedside examination. Patients who were admitted under General Surgery in both male and female wards from 1st of January till 16th of January at Queens Hospital Burton and were planned or indicated for DO monitoring were included. All patients from 18 to 90 years of age, who had emergency surgical admissions and were listed for elective colon cancer surgeries, were included. Those who underwent day case surgery were excluded from the study. Audit was registered with the audit department of the Trust. 


\section{Results}

Total 77 patients were included. Minimum age was 19 years and maximum age was 90 years. 34 were male patients and 43 were female patients. Among them only 8 patients had drain in situ during this period. Drain output was monitored in 7 patients (Figure 1). Only 4 patients had a plan in place for drain output monitoring (Figure 2).

\section{Discussion}

The use of surgical drains has been controversial over the years. The opinions in support of their use incorporate the fact that drains remove collected fluid, which is a likely source of infection; they protect against extra collections; they may allow initial recognition of anastomotic leaks or haemorrhage; leave a tract for percutaneous therapy and for latent collection to drain following subsequent removal. On the other hand, the existence of drains in the body raises the risk of infection; rises the duration of hospital stay; delays tissue healing; injury to the tissue caused by mechanical pressure or suction and drains may in fact provoke

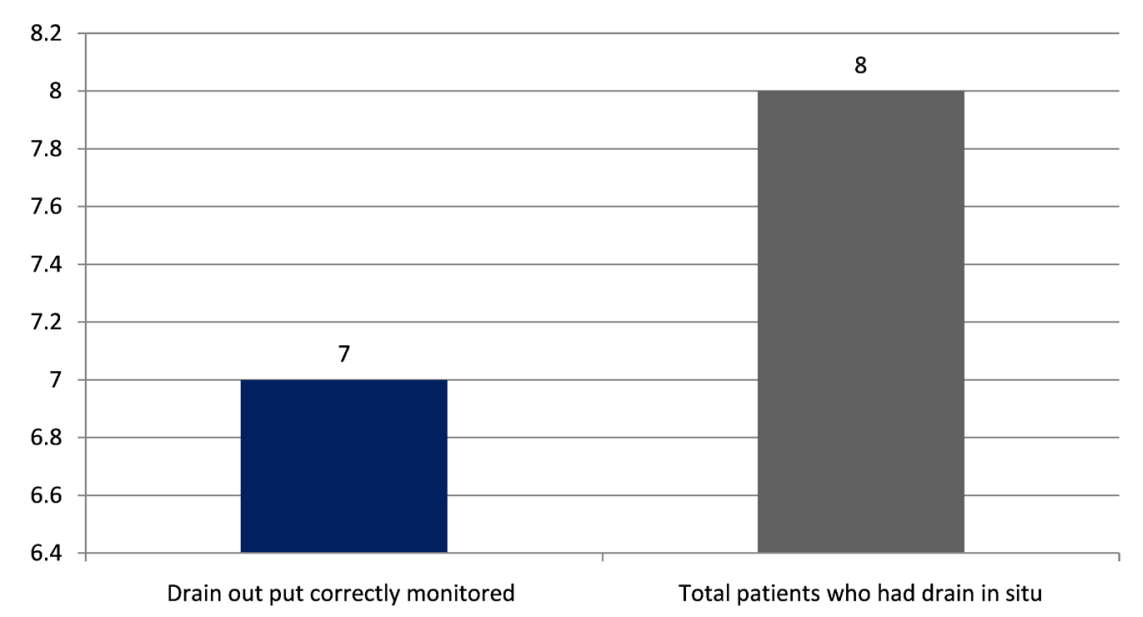

Figure 1. Bar diagram showing Drain output monitoring in indicated surgical patients.

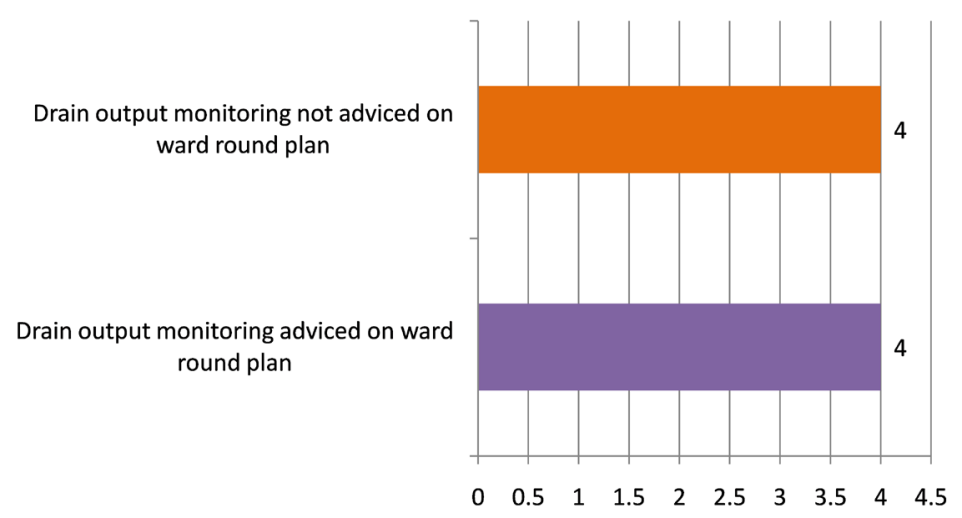

Figure 2. Number of patients instructed to monitor Drain output in ward. 
an anastomotic leak. These theories obviously are based on the hypothesis that there are no complications associated to the use of drains. Drains are now proven not to be harmless when left in situ. To drain or not, which drain and for how long all remain unreciprocated problems, but the multiplicity of answers suggests that no single policy is essentially correct. Every condition must be judged on its own merit, and the most suitable drainage approach and material judiciously selected. This strategy is more feasible than a rigid approach. A drain is believed to be misused when it is applied for the erroneous indication. Prolonged use of drain after it is due for removal has no extra benefit. Early removal of a drain before it has achieved its task raises morbidity, and misuse of resources. Incorrect choice of materials for an indication may be devastating. Improper placement of a drain makes it useless. Inadequately secured drain may dislodge. The use of considerably narrowed lumen tube drains is frequently futile and can certainly block. The use of drain to deliver a false sense of protection or as an alternative for sufficient haemostasis is harmful, and surgically objectionable. Failure to defend the drain from contamination by faeces, urine etc. enhances the frequency of surgical site infection. Kinking, knotting or blockage of surgical drains may lead to disaster. Drain must be passed across an individual stab wound and not through the main wound. A surgical drain position should be appropriate and remain efficient to maximize its impact.

\section{Conclusion}

Monitoring the output from surgical drains is important part of post-operative care. Appropriate drain output monitoring would improve patient safety, the efficiency of patient discharge and the stress of the surgical ward round. With better evidence, management of surgical patients should improve, and we should be able to practise based upon sound scientific principles rather than simply "doing what I always do". A written protocol can help staff on the ward with the aftercare of surgical drains.

\section{Disclosure and Ethics}

The authors have no conflict of interest to disclose. No funding was involved. This study was registered with the relevant department of the hospital. No intervention was carried other than the usual protocol and no patient identifiable data were used. Thus, patients were verbally consented.

\section{Conflicts of Interest}

The authors declare no conflicts of interest regarding the publication of this paper.

\section{References}

[1] Okada, N., Narita, Y., Takada, M., et al. (2013) Early Removal of Drains and the Incidence of Seroma after Breast Surgery. Breast Cancer, 22, 79-83. 
https://doi.org/10.1007/s12282-013-0457-3

[2] Barton, A., Blitz, M., Callahan, D., Yakimets, W., Adams, D. and Dabbs, K. (2006) Early Removal of Postmastectomy Drains Is Not Beneficial: Results from a Halted Randomized Controlled Trial. The American Journal of Surgery, 191, 652-656. https://doi.org/10.1016/j.amjsurg.2006.01.037

[3] Kim, H., Dykes, P.C., Thomas, D., Winfield, L.A. and Rocha, R.A. (2011) A Closer Look at Nursing Documentation on Paper Forms: Preparation for Computerizing a Nursing Documentation System. Computers in Biology and Medicine, 41, 182-189. https://doi.org/10.1016/j.compbiomed.2010.08.006 